\title{
Cardiac valve prostheses, anticoagulation, and pregnancy
}

\author{
MOHAMED BEN ISMAIL, FÉKRIA ABID, SALOUA TRABELSI, \\ MOHSEN TAKTAK, MANOUBIA FEKIH \\ From the Department of Cardiology, Ernest Conseil Hospital, Tunis, Tunisia
}

SUMMARY The course of 76 pregnancies is reported in 51 women who became pregnant after replacement of one or more heart valves. Age at conception ranged from 17 to 39 years (mean 25). There have been 71 deliveries and five women are still pregnant at the time of writing. In the 71 pregnancies, oral anticoagulants were given during 53, heparin during five, and no anticoagulants during 13. Fetal complications consisted of 12 spontaneous abortions (eight in pregnancies in which oral anticoagulants were given, three in which heparin was given, and one in which no anticoagulants were given), of 12 premature deliveries with seven stillbirths (three on oral anticoagulants and four without anticoagulants), and there were three neonatal deaths (in all three instances oral anticoagulants had been given during pregnancy). The maternal complications were as follows. Two women with mitral valve prostheses on heparin had thromboembolic episodes. Four women on oral anticoagulants died and 11 developed haemorrhage or systemic embolism. Two of the deaths were caused by bacterial endocarditis, one was the result of obstruction of a mitral valve prosthesis, and one was due to haemorrhage. One patient developed pulmonary oedema during delivery which rapidly resolved. Seven patients had uterine bleeding after delivery (three of them were on heparin and one was on an antiplatelet agent).

In 1966 DiSaia reported the first pregnancy and delivery in a patient with a Starr-Edwards valve prosthesis. ${ }^{1}$ Since then there have been numerous case reports and a few small series. A review of one of the largest series, comprising 25 women and 28 pregnancies, appeared in $1976 .{ }^{2}$ Complications of pregnancy are mainly related to the continuous use of anticoagulants. The increased coagulability normally seen in pregnancy increases the requirement for anticoagulants in women with heart valve prostheses. We report our experience of a large series ( 51 women, 76 pregnancies) and describe the difficulties associated with the management of pregnancy after heart valve replacement.

\section{Patients and methods}

There have been 76 pregnancies in 51 of 392 women aged $<40$ years with prosthetic heart valves who have been followed by us at this hospital. There have

Requests for reprints to Professor Mohamed Ben Ismail, Hôpital Ernest Conseil, Service Cardio 13, Tunis, Tunisia.

Accepted for publication 24 June 1985 been 71 deliveries in 47 women, and five women are still pregnant. Patients were aged from 10 to 40 (mean 23 years) at operation. Seven patients were aged less than 15 years. The interval between the operation and first pregnancy ranged from 4 months to 132 months (mean 42 months); two patients had heart valve replacement in the second and third months of pregnancy. Age at conception ranged from 17 to 39 years (mean 25 years). Sixty eight prostheses were inserted (53 Starr-Edwards, seven Björk-Shiley, four Lillehei-Kaster, and a xenograft in four). Twenty eight patients had the mitral valve replaced, seven the aortic valve, 15 had a double valve replacement (either mitral and aortic (10) or mitral and tricuspid (five)), and one patient had a triple valve replacement.

\section{PREOPERATIVE OBSTETRIC HISTORY}

Thirty three women had not been pregnant before operation for heart disease and seven had had an abortion, either induced (two) or spontaneous'(five). All but two of the pregnancies occurred without the woman first seeking medical advice. Fifty four pregnancies were reported in the first trimester, 18 in the 
Table Outcome of 71 pregnancies in women with cardiac valve prostheses

\begin{tabular}{|c|c|c|c|c|}
\hline & Heparin only & None & Oral anticoagulants & Total \\
\hline $\begin{array}{l}\text { Spontaneous abortion } \\
\text { Premature deliveries } \\
\text { Stillbirths } \\
\text { Neonatal deaths } \\
\text { Normal fullterm deliveries } \\
\text { Total number of pregnancies }\end{array}$ & $\frac{3}{-}$ & $\begin{array}{c}1 \\
4 \\
(4) \\
8 \\
13\end{array}$ & $\begin{array}{l}8 \\
8 \text { (two of those lived) } \\
(3) \\
(3) \\
37 \\
53\end{array}$ & $\begin{array}{r}12 \\
12 \\
7 \\
3 \\
47 \\
71+(10)\end{array}$ \\
\hline
\end{tabular}

^Figures in parentheses are already included in one of the events.

second trimester, and two in the third. Two pregnancies went to term without any follow up or treatment.

\section{ANTICOAGULANT TREATMENT}

Oral anticoagulants were given during 53 of the 71 pregnancies. The five women who are currently pregnant (three in the fourth month, one in the sixth month, and one in the eighth month) are also on oral anticoagulants. Heparin was used during five pregnancies. No anticoagulants were given in 13 pregnancies: in 10 of these oral anticoagulants were discontinued before pregnancy, in two they were changed to antiplatelet treatment during the second month of pregnancy, and in one an antiplatelet agent was given.

\section{Results}

\section{FETAL COMPLICATIONS}

In the five pregnancies in which heparin only was given, there were three spontaneous abortions in the second, third, and fifth months of pregnancy. The other two pregnancies were normal and went to term (Table). Of the 13 pregnancies without anticoagulant treatment, eight resulted in normal deliveries, four in stillbirths after premature delivery, and one in spontaneous abortion. In the 53 pregnancies in which oral anticoagulants were given, there were eight spontaneous abortions in the second, third, and fourth months of pregnancy; of the eight premature deliveries there were three stillbirths, three neonatal deaths, and two infants who lived. There were 37 fullterm normal deliveries in pregnancies in which oral anticoagulants had been replaced by heparin at the start of the ninth month. Thus, the 71 pregnancies resulted in 12 spontaneous abortions (three on heparin, one without anticoagulants, and eight on oral anticoagulants), in 12 premature deliveries with seven stillbirths (four without anticoagulants, three on oral anticoagulants), three neonatal deaths shortly after birth (all on oral anticoagulants), and two living infants; and 47 fullterm deliveries with only one death as a result of gastric haemorrhage (two on heparin, eight without anticoagulants, and 37 on oral anticoagulants). Twenty nine female and eighteen male babies survived. The median birthweight was $3 \mathrm{~kg}$ (range 2 to $3 \cdot 8$ ). Most of the surviving children are too young for progress in school to be assessed; however, none had detectable brain damage, mental retardation, or serious congenital abnormality.

\section{MATERNAL COMPLICATIONS}

Seven women had cardiac failure, but it responded to rest, salt restriction, digoxin, and diuretics. There were no haemorrhagic complications in the women on heparin, but two had thromboembolisms. Despite apparently regular and efficient anticoagulant treatment, one patient, aged 20 years, had thrombosis of a Starr-Edwards mitral valve prosthesis 24 hours after delivery of a normal child; reoperation was unsuccessful and the patient died in the immediate postoperative period of cardiogenic shock. Another patient with a Starr-Edwards mitral valve prosthesis had four thromboembolic episodes. The first, during the seventh month of pregnancy, resulted in right hemiplegia. The other three episodes occurred in the second and fourth month of pregnancy and on the sixth day after delivery. This patient was reoperated three weeks after the delivery of a fullterm, normal child. There were fibrin deposits at the base and the top of the prosthesis; three years before, this woman had had a coronary embolus when she temporarily stopped anticoagulant treatment.

There were no thromboembolic episodes in the pregnancies without anticoagulants. Two women had bacterial endocarditis. In one it was caused by Klebsiella; it occurred in the sixth month of pregnancy resulting in premature delivery. In the other endocarditis was caused by Candida albicans; it occurred a few days before delivery and was resistant to antifungals. The patient was reoperated two months after delivery and died from severe septicaemia.

There were 38 uncomplicated pregnancies in women on oral anticoagulants; four patients died, and 11 had complications (uterine haemorrhage and embolism) which rapidly resolved. Two deaths were caused by bacterial endocarditis 25 days and one month after delivery; one death was due to an 
obstruction of a Starr-Edwards mitral valve prosthesis a few days after delivery in a patient who was on oral anticoagulants until the beginning of the ninth month of pregnancy and was then on heparin. Heparin was stopped at the start of labour and restarted six hours after delivery. The fourth death occurred in the seventh month of pregnancy in a woman who had a severe haemorrhagic episode due to an excessive dose of anticoagulants; the fetus died in utero.

At delivery pulmonary oedema developed in one patient, this responded rapidly to treatment; seven patients presented with uterine bleeding after delivery: three of them were on heparin and one was on an antiplatelet agent.

\section{Discussion}

It is difficult to predict the outcome of pregnancy in women with artificial heart valves. Cardiac output increases by up to $50 \%$ in pregnancy; this is normally achieved by an increase in stroke volume rather than by an increase in heart rate. ${ }^{3}$ Like others, ${ }^{245}$ we found that pregnancy in women with heart valve prostheses, even those with triple valve replacement, is usually well tolerated when the heart has a good ventricular reserve, is in sinus rhythm, and has properly functioning valves. ${ }^{6}$

Increases in the concentrations of coagulation factors ${ }^{4}$ and a reduction in fibrinolysis in pregnancy ${ }^{7}$ add to the risk of thromboembolism associated with the presence of a prosthetic heart valve. The clinical manifestations of thromboembolism associated with artificial heart valves in pregnant women do not differ from those seen in non-pregnant women. ${ }^{8}$ The most serious complication that we saw was prosthetic valve thrombosis in three patients, which was fatal in two, despite reoperation in one case. Thrombosis of the artificial valve can be prevented by well controlled anticoagulant treatment. Discontinuation of oral anticoagulants or a change in the anticoagulation regimen in pregnant women, usually at the mother's request to reduce the risk to the fetus, may considerably increase the risk of maternal thromboembolism. In a review of 81 pregnant women with cardiac valve prostheses in which 24 patients stopped anticoagulants before or soon after conception, there were six patients with one or more thromboembolic episode and two deaths; whereas only three of the 57 patients on anticoagulants had embolic complications. ${ }^{9}$ In Limet and Grondin's series of seven patients one of three patients in whom anticoagulants were stopped before or early in pregnancy died of pulmonary oedema. Necropsy showed a recent friable thrombus engulfing the struts of the prosthesis. One of the four patients on anticoagulants who took their medications haphazardly had a periprosthetic thrombus which led to pulmonary oedema and a saddle embolus of the bifurcation of the aorta. ${ }^{9}$ Our results accord with those of Larrea et al who reported that thromboembolic complications were common in mitral valve prostheses. ${ }^{6}$ In their study of $\mathbf{3 8}$ patients in $\mathbf{4 7}$ pregnancies three $(7.9 \%)$ developed thrombosis of their mechanical valves-two Björk-Shiley mitral valve prostheses thrombosed 72 hours after delivery and one Björk-Shiley aortic valve prosthesis became thrombosed in the sixteenth week of pregnancy. ${ }^{6}$ All three thromboses occurred suddenly during apparently well controlled heparinisation, and without apparent change in the clotting time. Two of our five patients on heparin had thrombosis of a mitral valve prosthesis despite well controlled anticoagulation, and one of the patients on oral anticoagulants died of obstruction of a Starr-Edwards mitral valve prosthesis a few days after delivery: this patient was on oral anticoagulants until the start of the ninth month, she was then treated with heparin which was stopped at the start of labour and restarted six hours after delivery. Five other patients on oral anticoagulants had embolic episodes which rapidly resolved (one peripheral, one coronary, and three cerebrovascular). There were no thromboembolic episodes in any of our patients who were not on anticoagulant treatment during pregnancy.

It is widely believed that discontinuation of anticoagulant treatment increases the risk of systemic embolisation in the mother; on the other hand, their use during early pregnancy, near term and at delivery has been reported to be associated with an increased incidence of fetal malformation, stillbirths, and neonatal deaths from haemorrhage. Oral anticoagulants have small molecules that can cross the placenta and they are known to be teratogenic. In 1949, studies in rabbits by Kraus et al indicated that the use of coumarin during pregnancy may endanger the fetus. ${ }^{10}$ In 1966 DiSaia reported on the first pregnancy after implantation of a cardiac valve prosthesis; this fullterm infant, born to a mother who had received coumarin treatment in early pregnancy, had severe nasal hypoplasia and bilateral optic atrophy. ${ }^{1}$ Shaul and Hall summarised the abnormalities seen in 11 patients who had been exposed prenatally to warfarin derivatives. ${ }^{11}$ The most constant features were nasal hypoplasia and stippled epiphyses. ${ }^{11}$ Hall et al reviewed 24 cases in 1980 and confirmed these findings. ${ }^{12}$ Other abnormalities included optic atrophy, cataracts, mental retardation, and flexion contractures.

The critical period for teratogenesis is the sixth to the ninth week of gestation, and this may explain why we did not see malformations in our series. Our 
patients had not usually been treated with anticoagulants in the first trimester. Fetal loss due to abortion, stillbirth, or premature delivery is another complication of anticoagulant treatment. We and others have established that there is unquestionably an increased risk of fetal loss in pregnant women treated with anticoagulants. This risk is especially high when oral anticoagulants are administered from early pregnancy until term and when the dose of anticoagulant is sufficient to depress the prothrombin concentration even for only two days. ${ }^{91314}$ In a large review in 1975 Harrisson and Roschke noted that in 55 pregnancies in which the mother had been receiving an anticoagulant (usually warfarin) throughout pregnancy, fetal wastage was $29 \%$, whereas there was no fetal loss in 14 pregnancies in which no anticoagulants were used. ${ }^{15}$ Lutz et al reported spontaneous fetal wastage of $56 \%$ in pregnancies in which coumarin anticoagulants had been given in the first trimester. ${ }^{16}$ Ibarra-Perez et al reported nine spontaneous abortions in 25 pregnancies in which oral anticoagulants were used virtually throughout pregnancy. ${ }^{2}$ In 1979 we reviewed 133 published cases in which there was $30 \%$ fetal wastage ( 21 abortions and 18 stillbirths); of the 39 fetal losses, only one occurred in a patient who was not on anticoagulants and in 10 women there was an obviously reduced coagulability; 12 of the 18 perinatal deaths occurred when oral anticoagulants were taken until term. ${ }^{8}$

Hirsh et al stressed the importance of discontinuing oral anticoagulants before term to allow their elimination by the fetus and the restoration of normal prothrombin time. ${ }^{17}$ There is evidence that abortions, stillbirths, and early deaths are caused by placental detachment or internal or cerebral haemorrhage; bleeding may be due to the hypocoagulable state in the fetus, ${ }^{8}$ which is very sensitive to anticoagulants because of the immaturity of the liver. Thus the fetal prothrombin time may be considerably prolonged even when that of the mother is normal. ${ }^{18}$ To avoid bleeding complications in the fetus, maternal prothrombin times should be maintained in the low normal range. There is evidence that heparin does not cross the placenta and therefore it is unlikely to be teratogenic. ${ }^{18}$ Administration and monitoring of treatment are difficult, however, and fetal and maternal haemorrhage may occur. The maternal complication rate was high in a series of 135 cases. Fourteen patients $(10 \%)$ had a haemorrhage and three $(2 \%)$ of them died. ${ }^{12}$ Thirty three per cent of deliveries were premature or stillbirths. Heparin may be substituted for oral anticoagulants in pregnant women with heart valve prostheses, but this change can only be purposefully instituted when the pregnancy is recognised early and before the most vulnerable period for the fetus.

\section{Conclusions}

The risk of heart failure during pregnancy in women with heart valve prostheses is usually not great when bed rest and appropriate medical treatment are feasible; however, patients in functional class III and IV of the New York Heart Association classification with large left atrium and atrial fibrillation should be advised against pregnancy. Such patients are, however, not often encountered because most of them will have had surgical treatment before pregnancy in order to prevent deterioration of cardiac function.

The maternal risk of thromboembolism is high and it is not always prevented by anticoagulants. We found that heparin was less effective than oral anticoagulants and we did not find any teratogenic effects associated with oral anticoagulants. So, although many prefer to use heparin in the first trimester of pregnancy, we do not think that this is justified, even when pregnancy is diagnosed sufficiently early or is planned for. We do, however, recommend the use of heparin at the end of pregnancy to reduce the risk of haemorrhage during labour and delivery. Mothers on anticoagulants are often advised against breast feeding; however, there is evidence that those on warfarin may safely breast feed their infants. ${ }^{19}$

Because of the difficulties encountered in the management of pregnant women with artificial heart valves, some workers strongly recommend the use of bioprosthetic heart valves in women of child bearing age; but we believe that this attempt to reduce the risk of rapid valve failure is justified only in patients in sinus rhythm who are $>25$ years old.

\section{References}

1 DiSaia PJ. Pregnancy and delivery of a patient with a Starr-Edwards mitral valve prosthesis: report of a case. Obstet Gynecol 1966; 28: 469-72.

2 Ibarra-Perez C, Arevalo-Toledo N, Alvarez-de la Cadena $\mathrm{O}$, Noriega-Guerra $\mathrm{L}$. The course of pregnancy in patients with artificial heart valves. $A m \mathcal{F}$ Med 1976; 61: 504-12.

3 Oakley C, Doherty P. Pregnancy in patients after valve replacement. $B r$ Heart $\mathcal{F}$ 1976; 38: 1140-8.

4 Casanegra P, Avilés G, Maturana G, Dubernet J. Cardiovascular management of pregnant women with a heart valve prosthesis. Am $\mathcal{F}$ Cardiol 1975; 36: 802-6.

5 Taguchi K. Pregnancy in patients with a prosthetic heart valve. Surg Gynecol Obstet 1977; 145: 206-8.

6 Larrea JL, Núnẽz L, Reque JA, Gil Aguado $M$, Matarros R, Minguez JA. Pregnancy and mechanical valve prostheses: a high risk situation for the mother and the fetus. Ann Thorac Surg 1983; 36: 459-63. 
7 Moisson P, Cornier E, Rosensztajn L, Catinat J. Thrombose d'une prothèse valvulaire mitrale chez une femme enceinte. Traitement par la streptokinase. Coeur Med Interne 1979; 18: 425-32.

8 Ben-Ismail M, Fekih M, Taktak M, Chelli M. Prothèses valvularies cardiaques et grossesse. Arch Mal Coeur 1979; 72: 192-9.

9 Limet R, Grondin CM. Cardiac valve prostheses, anticoagulation, and pregnancy. Ann Thorac Surg 1977; 23: $337-41$.

10 Kraus AP, Perlow S, Singer K. Danger of dicumarol treatment in pregnancy. $\mathcal{F} A M A 1949 ; 139: 758-62$.

11 Shaul WL, Hall JG. Multiple congenital anomalies associated with oral anticoagulants. Am $\mathcal{F}$ Obstet Gynecol 1977; 127: 191-8.

12 Hall JG, Pauli RM, Wilson KM. Maternal and fetal sequelae of anticoagulation during pregnancy. Am $\mathcal{F}$ Med 1980; 68: 122-40.

13 Fillmore SJ, McDevitt E. Effects of coumarin com- pounds on the fetus. Ann Intern Med 1970; 73: 731-5.

14 Kronick J, Phelps NE, McCallion DJ, Hirsh J. Effects of sodium warfarin administered during pregnancy in mice. Am f Obstet Gynecol 1974; 118: 819-23.

15 Harrisson EC, Roschke EJ. Pregnancy in patients with cardiac valve prostheses. Clin Obstet Gynecol 1975; 18: 107-23.

16. Lutz DJ, Noller KL, Spittel JA Jr, Danielson GK, Fish CR. Pregnancy and its complications following cardiac valve prostheses. Am $\mathcal{F}$ Obstet Gynecol 1978; 131: $460-8$.

17 Hirsh J, Cade JF, Gallus AS. Anticoagulants in pregnancy: a review of indications and complications. $\mathrm{Am}$ Heart $f$ 1972; 83: 301-5.

18 Becker RM. Intracardiac surgery in pregnant women. Ann Thorac Surg 1983; 36: 453-8.

19 Orme ML'E, Lewis PJ, de Swiet M, et al. May mothers given warfarin breast-feed their infants? $\mathrm{Br}$ Med $\mathcal{F}$ 1977; i: 1564-5. 